\title{
Spatially resolved measurements of plasmonic eigenstates in complex-shaped, asymmetric nanoparticles: gold nanostars
}

\author{
S. Mazzucco ${ }^{1}$, O. Stéphan ${ }^{1}$, C. Colliex ${ }^{1}$, I. Pastoriza-Santos ${ }^{2}$, L.M. Liz-Marzan ${ }^{2}$, J. Garcia de Abajo ${ }^{3}$, \\ and M. Kociak ${ }^{1, a}$ \\ ${ }^{1}$ Laboratoire de Physique des Solides, Bâtiment 510, CNRS/UMR 8502, Univ Paris Sud 11, 91405 Orsay, France \\ ${ }^{2}$ Departamento de Química Física, and Unidad Asociada CSIC-Universidade de Vigo, 36310 Vigo, Spain \\ ${ }^{3}$ Instituto de Optica, CSIC, Serrano 121, 28006 Madrid, Spain
}

Received: 2 November 2010 / Received in final form: 28 February 2011 / Accepted: 17 March 2011 Published online: 14 June 2011 - C) EDP Sciences

\begin{abstract}
We report on spatially resolved electron energy-loss spectroscopy studies of optical modes in individual star-shaped gold nanoparticles. We studied different morphologies, ranging from a spheroid to well-developed nanostars. For each shape, essentially two groups of modes are appearing: the first one is localized around the core of the nanostars and has an energy slightly less than the quasi-static dipolar mode of a gold sphere (about $2.2 \mathrm{eV}$ ); in the second group, the modes are localized at the end of the nanostar tips, with varying energies depending on the geometry of each tip and with energy down to $1.2 \mathrm{eV}$. The localization of the tip modes is interpreted with the help of boundary element methods simulations.
\end{abstract}

\section{Introduction}

Localized Surface Plasmons (LSPs) are electron density oscillations at the surface of a sub-wavelength-sized nanoobject [1]. Parameters such as size, aspect ratio, the presence of a substrate, global and local modifications of the environment play an essential role in determining the behavior of LSPs: they must be taken in account when modeling the LSP physics. This involves several levels of description: from the simple quasi-static resonances in the Drude model to the relativistic frame of the full continuum dielectric model for an object with arbitrary shape. Thus, it is not straightforward to understand how one can determine the physics of LSPs in nanoparticles (NPs).

As an example, the energy of localized surface plasmons in thin $(4-6 \mathrm{~nm})$ silver triangular nanoprisms redshifts as their length increases. Such phenomenon could be naively attributed to retardation effects [2]. However this is not correct, because the typical size of those nanoparticles is smaller than the optical wavelengths in vacuum. The explanation of this behavior lies instead on the fact that the opposite faces of the prism do couple, leading to an aspect ratio-dependent redshift [3]. The plasmon modes are then standing waves, just like in optical nanoantennas [4-6]. In this example, varying a global parameter, the aspect ratio, affects the local behavior of LSPs (e.g., the energy of the modes, the intensity of which peaks very locally).

\footnotetext{
a e-mail: mathieu.kociak@u-psud.fr
}

Alternatively to these "antenna-like" objects, there are some kinds of NPs for which the LSP coherence length is smaller than their dimensions $[7,8]$. Here, the plasmonic modes are not influenced by what happens far from them. While, of course, the dielectric environment always plays an essential role, it is the local parameters, e.g., the curvature radius of the surface of the particle, rather than the global ones, e.g., the size, that control the physics of localized surface plasmons. By controlling the morphology of a nano-object it would then be possible to locally modulate its plasmonic behavior. Such nano-objects could then be tailored in order to control the characteristic LSP modes at a scale of, say, $10 \mathrm{~nm}$.

The distinction between locally and globally modified LSPs may be blurred in the case of greatly asymmetrical NPs, an example of which is given by gold "nanostars". As the name suggests, these particles consist of a nearlyspherical core with several tips protruding in all directions (these particles are also named "branched", "multi-pod", "star-shaped", "lumpy" or even "sea urchin-like"). These NPs present localized surface plasmons that are weakly coupled to each other and depend strongly on the local features of the NP itself.

Recently, gold nanostars have raised the interest of the scientific community. Their particular shape, plus the fact that they are made of gold (very stable and biocompatible), makes them suitable for several applications, including biosensing on live cells [9] or Surface Enhanced Raman Spectroscopy (SERS) detection of very low concentrations of molecules [10]. Nanostars with different 
symmetry levels, aspect ratios and number of tips have been synthesized by several research groups [9,11-14]. Their optical properties have been explored by means of many different experimental and numerical techniques such as UV-visible scattering [11,14], Rayleigh scattering in a dark-field microscope $[9,15]$, SERS $[10,13]$, Finite Difference Time Domain (FDTD) simulations [16], Boundary Elements Method (BEM) [10,14] and Discrete Dipole Approximation (DDA) calculations [9,11].

The conventional optical methods have some limitations in determining the spatial distribution of LSPs in individual NPs. For example, the experimental extinction spectra of an ensemble of nanostars showed a broad feature at about $1.70 \mathrm{eV}$ [14]. One can expect such a broad feature to encompass a wide spectrum of LSP modes that are lost in the ensemble measurement which gives, in fact, an averaged signal. These modes may arise from the small differences that the NPs have among one another due to the lack of complete control of their synthesis. It is thus of the greatest interest of being able to map the LSPs of nano-objects with high-spatial resolution, i.e., at least $10 \mathrm{~nm}$.

Electron Energy-Loss Spectroscopy (EELS) in a Scanning Transmission Electron Microscope (STEM), in the following referred to as STEM-EELS, allows one to combine the optical information given by EELS [17] and the spatial resolution (down to $1 \mathrm{~nm}$ or even better) of a STEM. In the past decades, an important number of spatially resolved EELS studies in the low-loss region (where optical transitions are measured) have been performed; for a review, see, e.g., $[18,19]$. Most of these experimental and theoretical studies focused on the measurement of Interface Plasmons (IPs) and, to a smaller extent, to other boundary-related excitations for different geometries such as spheres [20], cylinders [21-27] and single or multi-layers [28-31]. However, they essentially considered both an energy range above $4 \mathrm{eV}$, i.e., the UV spectrum, and highly symmetric configurations. That was due both to a lack of combined high-spatial and high-energy resolution and of relevant theories or simulation tools for handling arbitrarily-shaped nanostructures. Recently, both limitations have been overcome [32-36] enabling the study of the optical properties of arbitrarily-shaped metallic nanoparticles at the nanometer scale.

For non-symmetric objects, no analytical formulation of the optical properties or of the electron energy losses exists so far: only numerical calculations can help supporting the experimental data.

In this letter, we present a systematic spatially resolved EELS study of gold nanostars. Different shapes have been considered during this work: the behavior of localized surface plasmons has been studied from the simple nearlyspherical shape to more complex multi-tipped particles. We demonstrate by direct measurements the existence of two types of modes: one corresponding to the core and the other to the different tips, which validates previous deductions based on averaged optical measurements. The core energy is close to that of a sphere, while each tip develops its own very localized plasmon at different energies. This argues for a high localization and clear absence

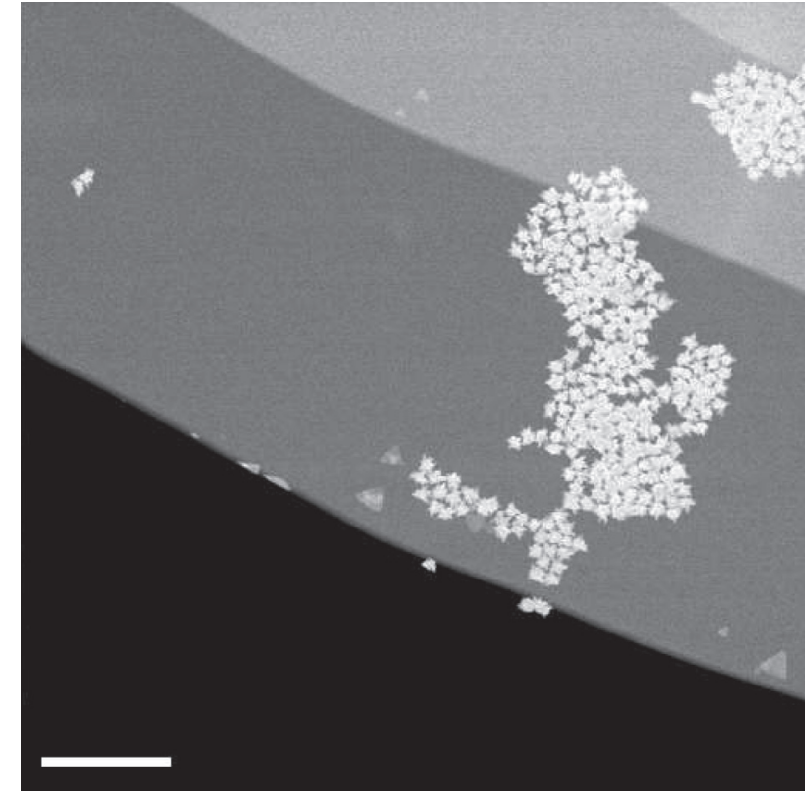

Fig. 1. HAADF image at low magnification showing a group of nanostars on cleaved mica. Scalebar is $500 \mathrm{~nm}$.

of cross-talk between the different tip modes. This result should have a direct consequence in the optimization of the design of single molecule SERS experiments.

\section{Experimental method and material}

The synthesis of the nanostars analyzed in this paper has been described elsewhere [14]. The samples have been prepared for STEM-EELS analysis as follows: a few drops of the colloidal solution of gold nanostars in ethanol were dropped on a cleaved mica sheet glued on a microscopy $\mathrm{Cu}$ grid, the grid was then allowed to dry for several minutes before the STEM-EELS experiments. During the STEM observations, the nanoparticles were often found aggregated in clusters, but it was not unusual to find isolated nanostars (Fig. 1). The observed particles were found to have a quite irregular morphology, their shapes ranging from nearly-spherical to well-formed nanostars (Fig. 2).

Spatially resolved EELS experiments have been performed on a VG HB501 STEM fitted with a GATAN 666 spectrometer, a custom, optically coupled CCD camera detection system and a custom scanning and spectral imaging unit. Such a system, operating at an acceleration voltage of $100 \mathrm{kV}$, allows one to form an electron probe of less than $1 \mathrm{~nm}$ onto the area of interest. After interaction with the sample, the electrons scattered at relatively high angles are gathered into a High Angle Annular Dark Field (HAADF) detector, which gives a signal roughly proportional to the projected mass density under the beam. Simultaneously, the electrons scattered at lower angles are collected onto the EELS CCD camera, giving rise to an Electron Energy-Loss (EEL) spectrum. After a suitable area of the sample is selected, e.g., an individual nanostar, the electron energy-loss spectra are acquired using the 


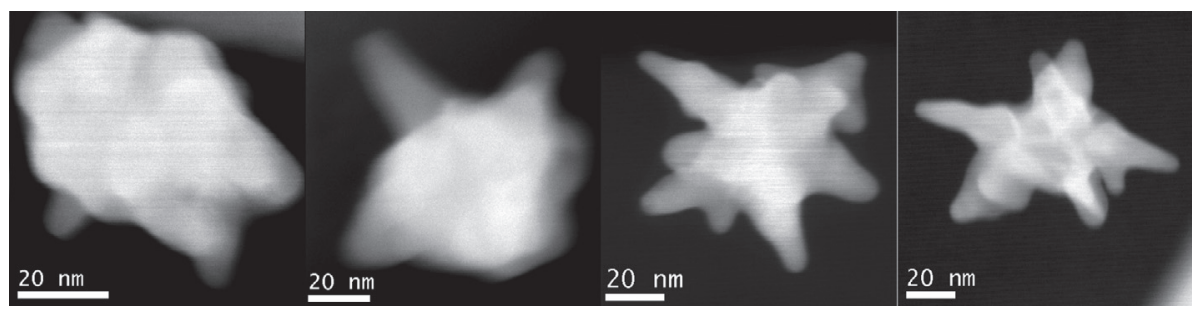

Fig. 2. Conventional (off-line) HAADF images of the studied nanostars, from left to right: S0, S1, S2 and S3. The complexity of the morphology increases moving from a spheroidal particle to well-formed nanostars.

Chrono Spectrum Image (ChSPIM) technique, a modification of the conventional Spectrum Image mode [37]. In this mode, the electron probe scans the sample with a constant spatial increment $(1-4 \mathrm{~nm})$ covering a total surface of typically $32 \times 32$ pixels. At each pixel, typically 50 energy-loss spectra are recorded in a spectral range below $80 \mathrm{eV}$ (low-loss range). The nominal energy dispersion during the experiments was $0.05 \mathrm{eV} /$ channel and the acquisition time was 2-3 ms per spectrum; the short acquisition time allows one to partially remove the instabilities due to the mains (power line) frequency $[36,38]$. In parallel to the ChSPIM, an "on-line" HAADF image was also acquired. The on-line HAADF makes possible to compare directly the relationship between the EEL spectra and the morphology of the NP. Right after the data acquisition, a series of 50-100 spectra in vacuum was recorded with the same experimental parameters; they were used to obtain the Point Spread Function (PSF) for the further deconvolution process.

During the off-line data treatment, each set of EEL spectra was then aligned along their Zero-Loss Peak (ZLP) - that is the peak for which the electrons have been elastically scattered - and summed to obtain a better Signal-to-Noise Ratio (SNR). In order to improve the visibility of the low-loss features, the summed spectra were deconvolved with the Richardson-Lucy (RL) algorithm [39]. Five iterations have been used for the RL deconvolution of the data. The dataset finally obtained can be thought as a cube whose axes are the two spatial dimensions and the energy loss, each point inside the cube contains the intensity of the EEL spectrum at a given position of the probe and at a given electron energy loss.

The resonance peaks below $3 \mathrm{eV}$ in the EEL spectra are the signature of the localized surface plasmons. The LSPs features were mapped using semi-automatic custom software procedures written for the commercial data analysis software Igor-Pro (from Wavemetrics Inc.) [40]. This set of procedures, especially developed for this aim, has been named EELSLL and is available on request [41]. EELSLL allows one to easily normalize the spectra, subtract the ZLP, identify and fit the plasmon peaks (with either a Gaussian or Lorentzian function, plus different background models) of the whole dataset. EELSLL also allows one to manipulate and display the parameters of the fit, e.g., the energy position of the peaks, their intensity and width, in maps where each pixel represents the value of the given parameter. It is by means of such maps that one can understand the behavior of LSPs at the nanometer scale.

\section{Experimental results}

Although the results found in the literature give a good picture about the physics of LSPs in gold nanostars, no direct local measurement of the plasmon modes has been reported so far. STEM-EELS offers the opportunity to perform such measurements with nanometric spatial resolution.

In order to better understand the role of the symmetry and whether the plasmonic resonances were influenced by local or global parameters, nanostars with shapes of increasing complexity were studied. Figure 2 shows the conventional (off-line) HAADF images for the selection of NPs that have been chosen for this publication, for the sake of clarity, a code-name has been assigned to each nanostar: S0 is the spheroidal particle, S1 is the "quasistar", S2 and S3 are well-formed multi-tipped stars.

Figure 3 summarizes the experimental results: the topto-bottom rows show the on-line HAADF image - that is, the image acquired simultaneously to the EEL spectra - the calculated relative thickness, the energy and intensity maps of the LSP modes with lowest energy, for S0, S1, S2 and S3 respectively. The spatial distribution of the higher energy mode for S2 and S3 is displayed in Figure 4. The black profile line has been extracted from the on-line HAADF images and is given as a reference. The differences between the on-line and off-line (conventional) HAADF images for stars S0 and S1 in Figures 2 and 3 are due to the sample drifting during the acquisition of the ChSPIM. However this does not affect the quality of the results sensibly.

Figure 5 shows EEL spectra in the low-loss range extracted from different areas (pixels) of the studied nanostars, each spectrum is detailed below. Finally, Figure 6 shows the energy histograms of the modes of the four selected nanostars, providing an overview of the energy distribution of the LSPs.

\subsection{S0: spheroid}

Particle S0 is a spheroid with an average core diameter of about $39 \mathrm{~nm}$. It hangs in vacuum from a piece of mica substrate visible in both the on-line HAADF image and relative thickness map. 

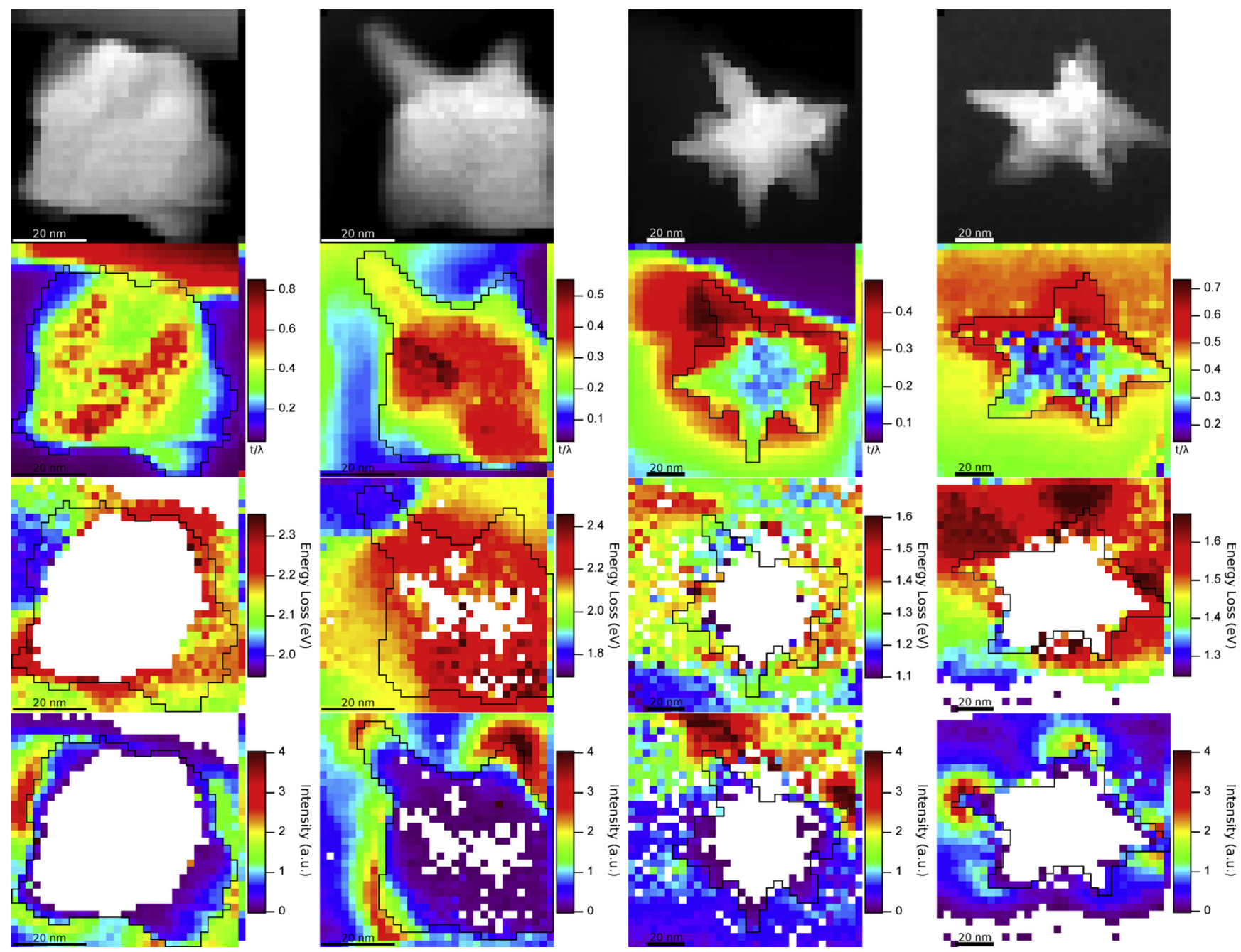

Fig. 3. (Color online) Summary of data on the analyzed particles. From left to right: S0, S1, S2, S3. From top to bottom: on-line HAADF image, relative thickness map $(t / \lambda)$, energy map for the low-energy LSP, intensity map for the low-energy LSP. The black line in the maps is taken from the on-line HAADF images. The white pixels in the maps indicate that the fitting procedure was not valid; this was likely due to a poor SNR in the spectrum. A detailed description is given in the main text.

Only one main plasmon mode of energy ranging from about $2.0 \mathrm{eV}$ to about $2.3 \mathrm{eV}$ is detected in $\mathrm{S} 0$. The average energy of the plasmon resonance is $2.15 \mathrm{eV}$. In a small zone of the particle, in a range of about $8 \mathrm{~nm}$, the plasmon energy reaches its minimum value of about $2.0 \mathrm{eV}$, the same zone also shows the highest plasmon intensity. By comparing the energy and intensity maps (Fig. 3), one can see that the regions of the particle where the energy is lower exhibit a higher intensity.

\subsection{S1: quasi-star}

Particle S1 has a degree of complexity greater than that of S0. Three tips protrude from the spherical core, the longer one lies on a thin mica substrate (visible only in the relative thickness map) whose thickness rapidly decreases. The core diameter is about $50 \mathrm{~nm}$, the longer tip is about $34 \mathrm{~nm}$ while the shorter tips measure about $20 \mathrm{~nm}$.

Because of its quasi-spherical core with two short tips and a longer one, particle S1 has been defined a "quasi- star". Despite its shape being still rather simple, the presence of the tips gives rise to supplementary LSP modes with respect to the case of the spheroid S0. The highenergy mode at the core of the particle lies at about $2.2 \mathrm{eV}$. Besides this mode, additional LSP resonances are detected at each tip of the nanostar. The longer tip, that lies on a thin mica substrate, has a resonance at $1.8 \mathrm{eV}$; the energy of the mode associated to the two shorter tips is instead $2.1 \mathrm{eV}$. Also, the highest LSP intensity is measured at the tips, where the modes are 3 to 4 times stronger than the core mode.

\subsection{S2: multi-tipped star, first example}

Particle S2 is a well-formed nanostar with five long tips visible in the image plane; each tip along the left-right direction of the HAADF image presents two supplementary "sub-tips". Other tips might be present on the central core in the direction parallel to the electron beam. They could 

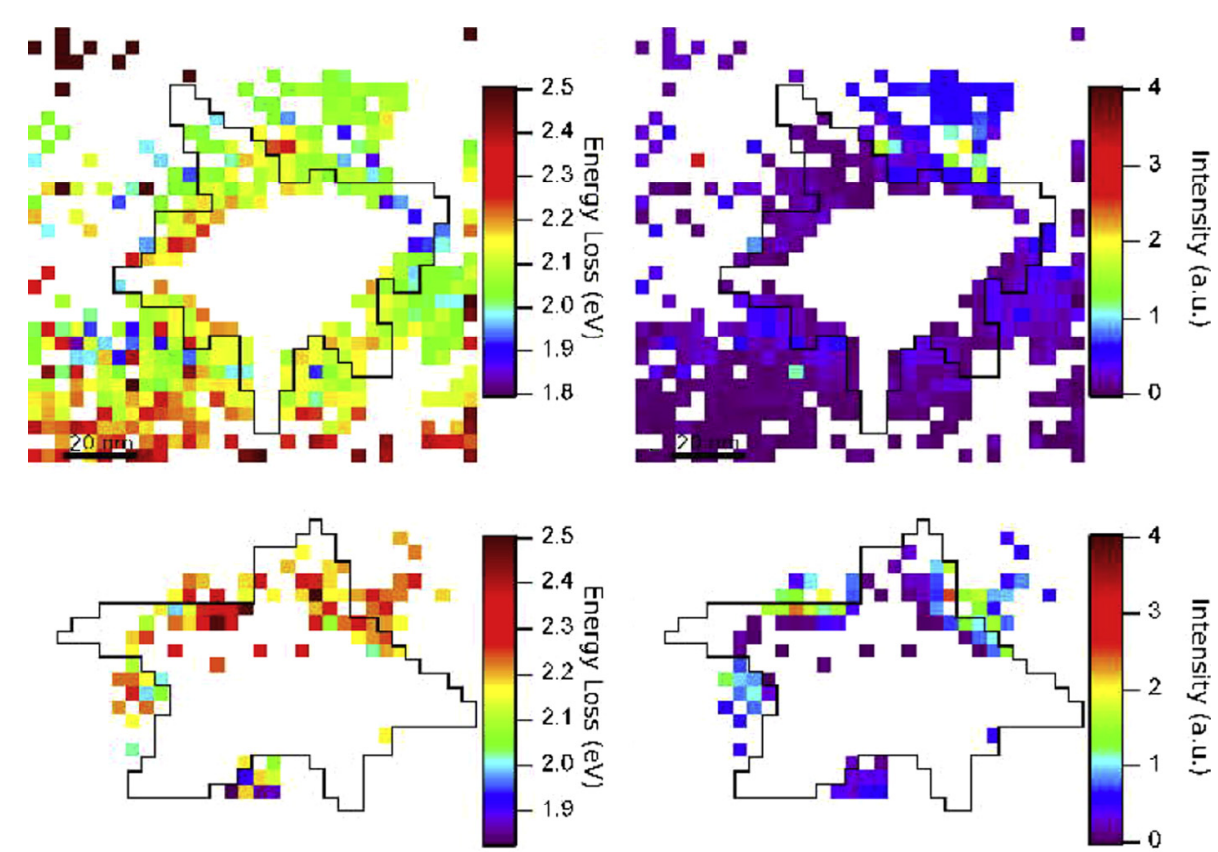

$\underline{20 \mathrm{~nm}}$

$-20 \mathrm{~nm}$

Fig. 4. (Color online) Energy and intensity maps for the core LSP mode in S2 and S3.

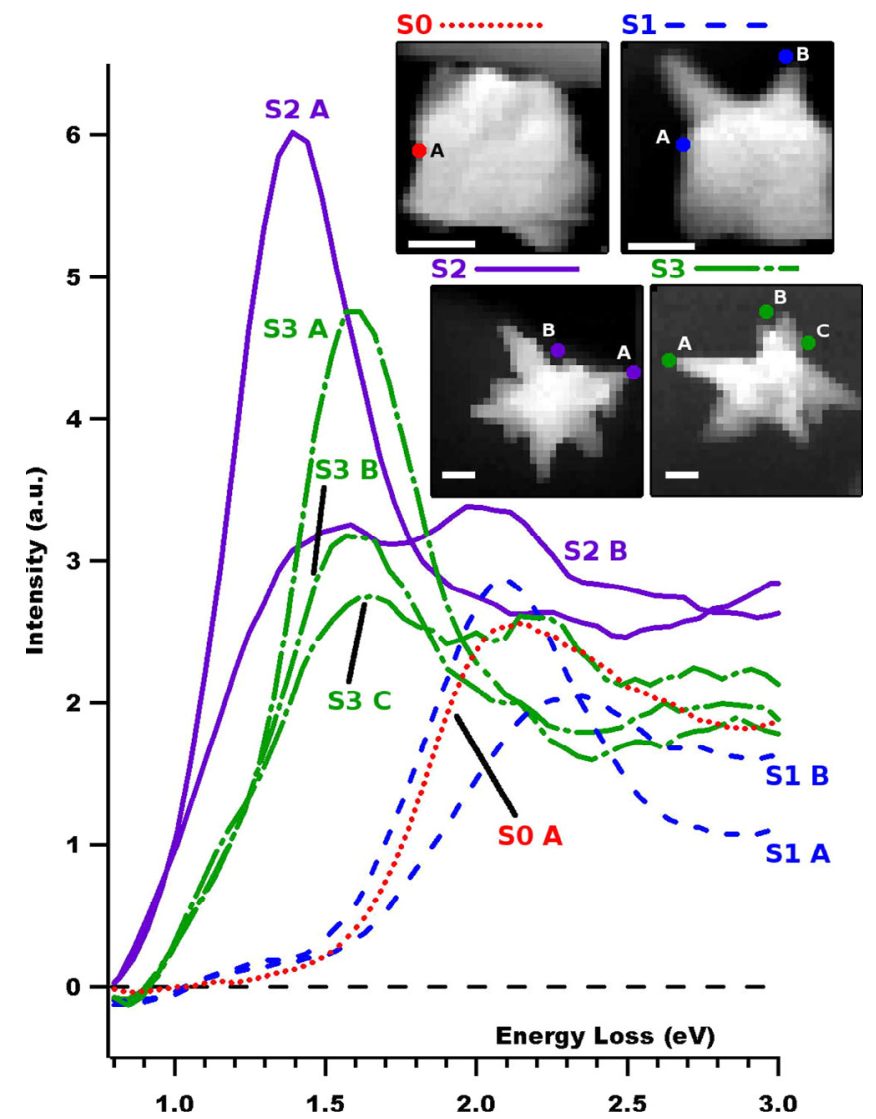

Fig. 5. (Color online) Electron energy-loss spectra extracted from given pixels (see inset) of the studied nanostars. be responsible for the high diffraction that results in extremely poor energy-loss signal in the core of the particle: this yields invalid values for the relative thickness map. The nanostar lies completely on mica but it is positioned near its border with vacuum; this is visible in the relative thickness map (Fig. 3). The core of the particle measures about $40 \mathrm{~nm}$ and the tip length is about $25 \mathrm{~nm}$.

In particle $\mathrm{S} 2$, the modes associated with the tips are prominent. Nevertheless, it has been possible to fit the high-energy core mode as well (spectrum S2B in Fig. 5). Figure 4 shows the energy and intensity maps for the highenergy modes. The apex of the longest tip presents a LSP at about $1.4 \mathrm{eV}$, while the resonances localized at the remaining tips have slightly lower energy at about $1.35 \mathrm{eV}$. The mode with highest energy is distributed around the core of the particle and it is peaked at about $2.1 \mathrm{eV}$. Particle $\mathrm{S} 2$ is lying a few nanometers away from the border between mica and vacuum, as shown by the relative thickness map in Figure 3. The intensity of the LSPs is higher in vacuum than on the mica substrate. However, one can still note that the modes are stronger at the tips.

\subsection{S3: multi-tipped star, second example}

Particle S3 is another multi-tipped nanostar, with five tips about $30 \mathrm{~nm}$ long, a sixth tip is directed either inward or outward the image plane as shown by the HAADF images in Figures 2 and 3; the core diameter is about $48 \mathrm{~nm}$. As for particle S2, the energy-loss signal inside S3 was poor due to diffraction effects so that the relative 


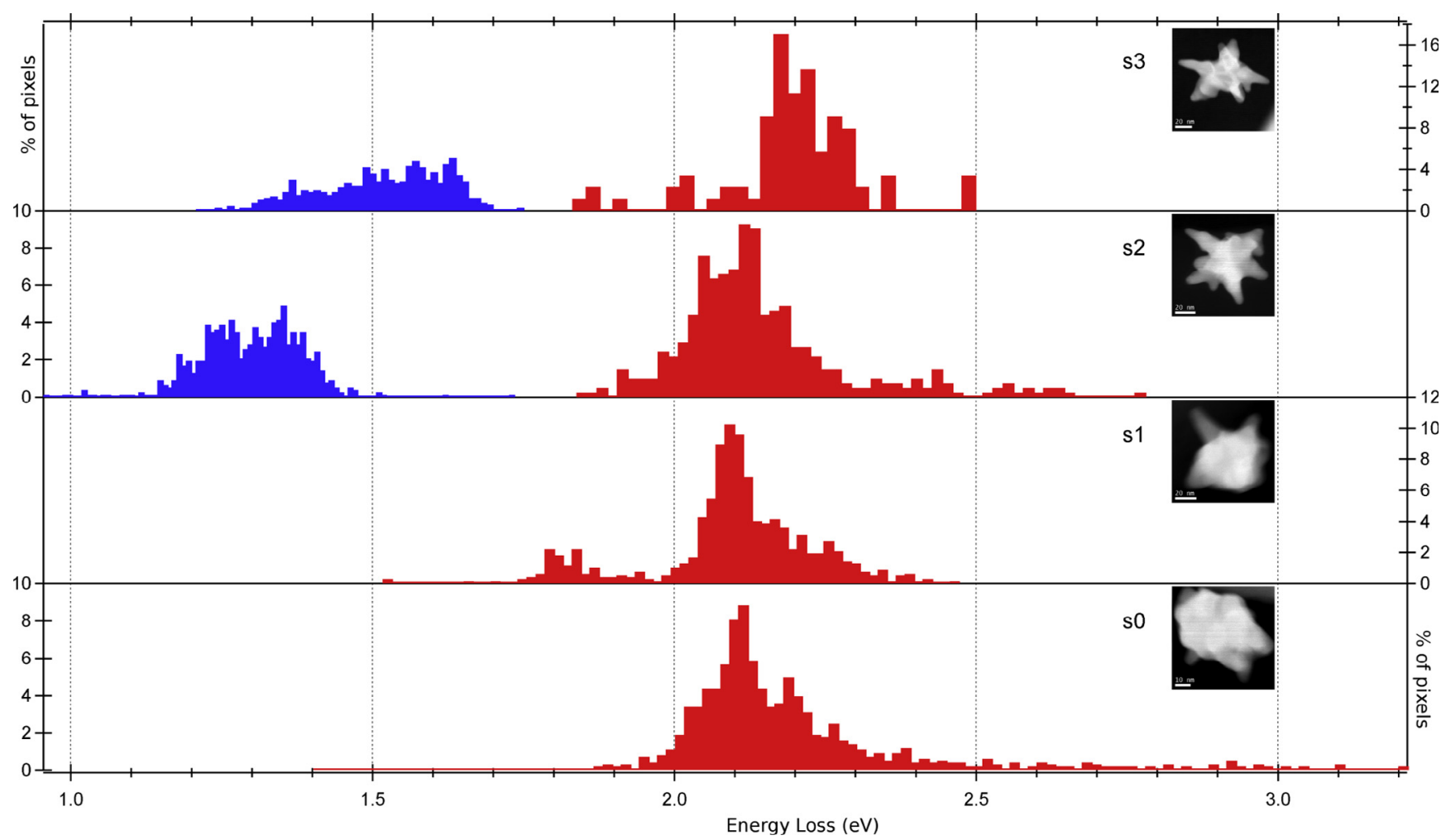

Fig. 6. (Color online) Histograms representing the percentage of pixels within a certain energy in the energy maps of the studied nanostars S0, S1, S2 and S3. For particles S2 and S3, the histograms calculated from the low- and high-energy modes are represented in blue and red respectively. A detailed description is given in the main text.

thickness map gave invalid values. This nanostar lies on the mica substrate.

Sample spectra acquired in correspondence of the tips and the core of the nanostar are shown in Figure 5. At the tips of the nanostar, LSP modes at about $1.6 \mathrm{eV}, 1.5 \mathrm{eV}$ and $1.35 \mathrm{eV}$ are detected. The mode associated with the core is also present at about $2.2 \mathrm{eV}$, but it is very weak and it is detected only at few pixels within the core. The maps displaying the energy position and the intensity of the plasmon resonances are shown in Figure 4.

\section{Discussion}

An instructive picture of the LSP energies is given by the histograms represented in Figure 6. The percentage of pixels within a given energy was calculated and a histogram was generated for each energy map.

For particle S0, the majority of the pixels are centered around $2.1 \mathrm{eV}$, but two smaller maxima are also present at about $2.2 \mathrm{eV}$ and about $2.3 \mathrm{eV}$ clearly reflecting the behavior seen in the energy map of Figure 3. The small difference in energy for this mode could be due to the local change of curvature on the particle surface.

For particle S1, the core mode is at about $2.2 \mathrm{eV}$, but the most important feature is at about $2.1 \mathrm{eV}$. This feature is due to the end of the shorter tips. These tip ends are also rounder than the longer one. The tip mode due to the longer tip is also clearly distinguishable at about $1.8 \mathrm{eV}$; the length and the sharpness of this tip could be respon- sible for this energy value. However one cannot exclude the possibility that this mode could be further redshifted because of the contact of the tip with the mica substrate.

Finally, two histograms are shown for each of the particles S2 and S3. The histograms relative to the core mode confirm again that this mode resembles the surface plasmon of a sphere. The histograms relative to the tip modes show that for particle S2 the overall energy of the resonances is lower than the energy of the LSP of particle S3.

All the nanostars present two kinds of LSP modes. The first one is found along the core of the particle and its energy is about $2.2 \mathrm{eV}$. In the classic dielectric continuum model, the dipolar polarizability of a sphere surrounded by vacuum in the dipole approximation diverges when the dielectric function $\epsilon\left(\omega_{l=1}\right)=-2$. From experimental optical data [42], the value of $\omega_{l=1}$ for gold is $\omega_{l=1}^{\mathrm{Au}}=2.56 \mathrm{eV}$. This is in agreement with the experimental measurements if one considers that the cores of the particles are not perfectly spherical, that an interaction with the mica substrate could redshift the energy of the mode and that, in general, elongation shifts the plasmon modes to lower energies.

The redshift of the core mode with respect to a sphere can also be explained by considering a geometrical effect [14]: the LSP induced charges can move easily along the surface of a sphere. The presence of the tips is an obstacle for these charges, thus the oscillation period of the core plasmon increases with a subsequent energy decrease. 
The maps and spectra also show that the ends of each tip in the nanostars are characterized by different energies, ranging from about $1.3 \mathrm{eV}$ to about $1.8 \mathrm{eV}$. These values are in agreement with the literature [16]. The second kind of plasmon resonance can then be associated with the tips of the nanoparticles. We note that, generally speaking, the tendency is a decrease of the energy of the tip end mode with increasing tip length, independently of the nanostars diameter, which is almost constant between the different nanostars.

The tips are characterized by LSP modes that are highly focused on their ends as shown by the intensity maps (Fig. 3). This means that the penetration of the tip plasmon mode inside the particle is weak and does not have a strong influence on the charge oscillations at the core or at the other tips; for this reason, the coupling between different tips in a single particle is weak. Furthermore, the probability of tip-to-tip coupling is decreased because the large dispersion in tip shape makes it unlikely that two identical tips sit together in the same particle. Moreover, the skin depth of gold at optical frequencies is about $15 \mathrm{~nm}$; then, it would be difficult for the electromagnetic field to pass through the whole particle.

In order to gain insight into the plasmon excitations involved, we performed BEM simulations of the EELS probability for electrons passing near the tip and near the center of model particles, and for other optical properties like extinction cross-section and field enhancement. The BEM method consists in expressing the exact solution of Maxwell's equations in the presence of arbitrarilyshaped dielectrics in terms of surface-integral equations evaluated at the interfaces. The electromagnetic field induced by the passage of an external electron modeled as a point charge, or a electromagnetic plane wave illumination, is then calculated in terms of self-consistently obtained boundary charges and currents. Electronic (EELS) and optical (extinction) spectra can thus be calculated for arbitrary shapes [33]. The model particles were chosen to reflect the salient features of the nanostars. Thus, we designed nanoparticles shapes having two tips separated by a core that we mold in the form of a semi-elliptical ring. The radius of curvature and the cone angle of the tips were chosen close to experimental values, while the ring parameters were varied. Notice that the total height (tipto-tip distance) is kept constant and the tip height does change on the right panel of Figure 7. On the other hand, on the left panel both total height and the tip height are kept constant, but the core is expanding laterally. This permits us to understand how the plasmons evolve as the tip size and core diameter are changing. For the electron passing near the tip, a prominent low-energy tip plasmon is observed, which is in excellent agreement with the extinction cross-section and the near-field enhancement at the tip, also shown in Figure 7 for light incidence with polarization along the particle symmetry axis. The interaction between the two tips in the particle is known to be negligible [14] because they are physically well far apart. These model particles represent reasonably well the actual samples, in which one can find tips of very different shape in each particle. Thus, inter-tip interactions play a minor role in the LSP behavior. The model calculations also provide useful insight into the effect of the rest of the particle to whom the tip belongs: the tip piles up induced charges in its apex, giving rise to the noted low-energy plasmon, and also generating large field enhancements; the rest of the particle acts as a charge reservoir, the influence of which depends on its actual shape, as shown in Figure 7.

When the tip length is kept constant, but the central part of the particle is made thicker (left column of Fig. 7), the tip plasmon moves slightly to higher energies, until it saturates at $\sim 1.4 \mathrm{eV}$ for an extra thickness $a \approx 10 \mathrm{~nm}$ (see upper scheme). This was expected as an indication that the tip plasmon is indeed mainly confined to the end of the tip. Interestingly, and in contrast to the optical simulations, the electron passing near the end of the tip picks up a second, higher-energy plasmon at $\sim 1.7 \mathrm{eV}$. This new plasmon dominates the EELS spectrum for electrons passing near the particle center, and its energy is situated in between the tip plasmon and the transversal (core) plasmon at $\sim 2.3 \mathrm{eV}$.

When the overall radius of the particle is kept constant but the central part is made thicker (right column of Fig. 7), the tip plasmon evolves toward higher energies as a consequence of the decrease in the length of the tip (this is because the total height of the particle is kept constant at $90 \mathrm{~nm}$ ). Eventually, one ends up with a rod-like particle with short tips. This clearly shows that the adiabatic thinning toward the end in the shape profile of an elongated tip is important to produce low-energy plasmons. These results are fully consistent with our EELS measurements and explain the evolution of the spectra from thicker particles with shorter tips (sample S0) to longer tips with similar particle size (samples S2 and S3).

\section{Conclusions}

Localized Surface Plasmons on individual star-shaped gold nanoparticles have been measured directly by STEM-EELS and mapped with nanometric resolution. A sphere-like core mode and several tip modes have been identified within the nanoparticles. Boundary Elements Method simulations strongly support the experimental results.

A clear measurement of the core LSP mode in nanostars has been demonstrated. The core excitation being 3 to 4 times weaker than the tip ones, its signal is overwhelmed by the tip resonances; it is thus very hard to detect such weak core LSP. The core mode is not detected even using Rayleigh spectroscopy of single nanostars [15], nor it could be with standard optical techniques, which probe over a vast population of nanoparticles and can only measure an averaged signal. Thanks to the high-spatial resolution of STEM-EELS and to advanced data treatment procedures, this mode has now been identified and mapped on individual nanostars.

LSP maps also show that each tip carries its own energy as already suggested by indirect measurements [15] and FDTD simulations [16]. STEM-EELS mapping allows 

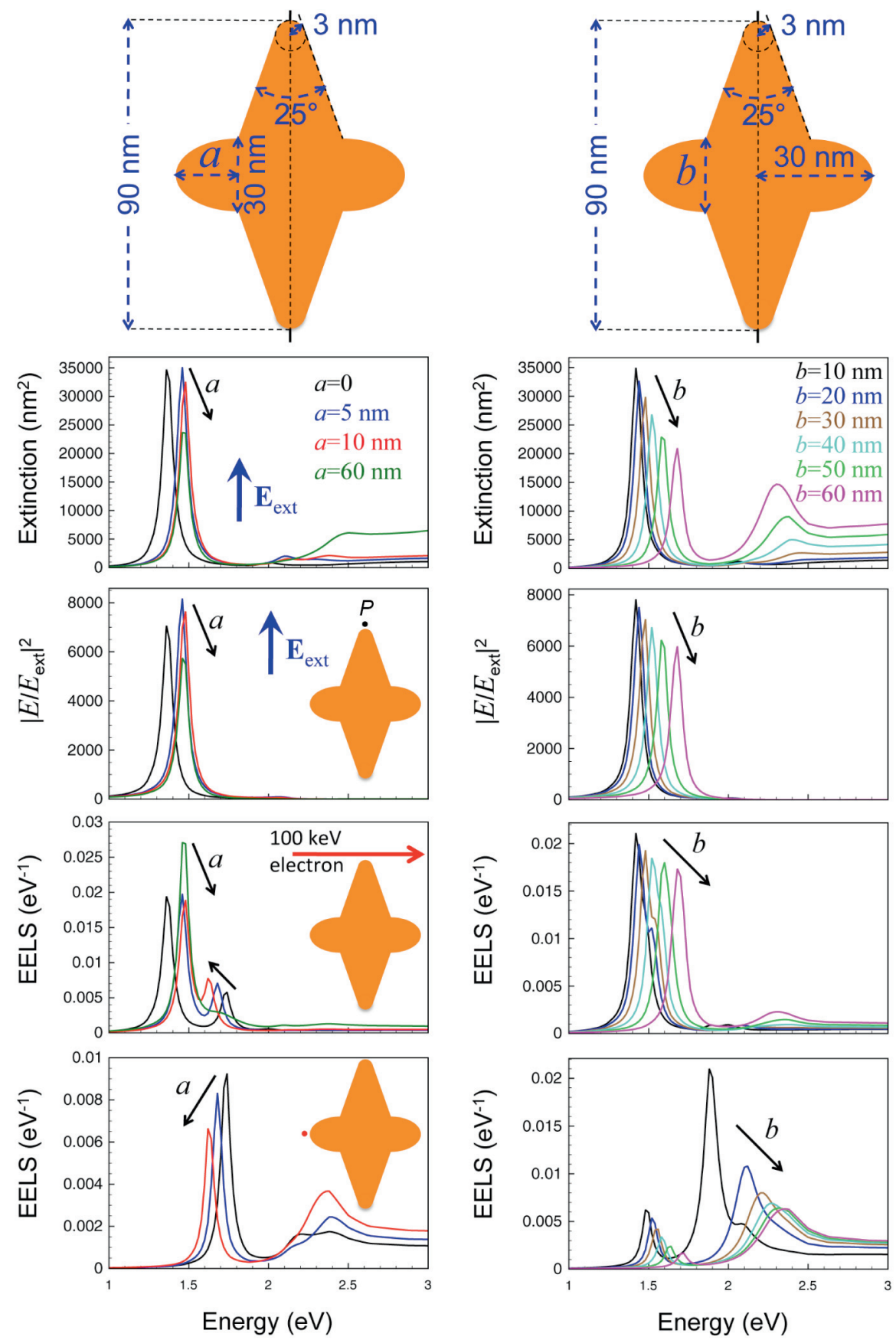

Fig. 7. (Color online) EELS, field enhancement and optical extinction simulations of tip plasmons in nanostars. For the EELS, the trajectory of the electron is notified by an arrow. For the field enhancement, the excitation polarization direction is indicated by an arrow, and the electric field is calculated at the point $P$. For the extinction, the excitation polarization is indicated by an arrow. We consider an axially symmetric particle with two tips, as shown in the upper schemes. The tip radius (3 nm), the tip angle $\left(25^{\circ}\right)$, and the total particle length $(90 \mathrm{~nm})$ are adjusted to best represent sample S2. The particle has a central disk joining the base of the tips and it is supplemented by a semi-elliptically-shaped ring protuberance of varying dimensions. Left: particles with a protuberance of $30 \mathrm{~nm}$ height and varying transversal size $a$, as shown in the upper-left scheme. Right: particles with varying protuberance height $b$ and fixed transversal size equal to $30 \mathrm{~nm}$, as shown in the upper-right scheme. The optical extinction and the electric field intensity at point $P$ are shown for light incidence with the polarization along the symmetry axis. The EELS probability is calculated for a trajectory passing close to the tip and for a trajectory passing near the particle surface at its center (see insets in the plots). The distance from the electron beam to the particle surface is $2 \mathrm{~nm}$ in all cases. Point $P$ for the near field is $2 \mathrm{~nm}$ away from the tip. All spectra are calculated using the Boundary Element Method (BEM) [7], with the gold represented by a dielectric function taken from optical data [43]. 
one to distinguish among the tip modes by means of a direct measurement of their energy, thus demonstrating that these resonances are different eigenstates characteristic of each tip.

Finally, each tip's mode depends on global (length) and local (tip's end curvature) parameters. However, the tip-to-tip interaction is weak due to the screening of the tips by the core, so that the global shape of the nanostar hardly influences the tip's LSP properties.

We would like to thank P. Senthil Kumar for help in nanoparticles synthesis. The authors acknowledge financial support from the European Union (E4) under the Framework 6 Program under a contract for an Integrated Infrastructure Initiative (reference 026019 ESTEEM), EU-FP6 (NMP4-2006-016881SPANS) and Marie Curie Program (MEST CT 2004 514307). They also acknowledge the support of the Agence Nationale de la Recherche under the project HYNNA.

\section{Disclaimer of endorsement}

Reference herein to any trademark, proprietary product, or company name is intended for explicit description only and does not constitute or imply endorsement or recommendation by the authors or anyone else.

\section{References}

1. E. Hutter, J.H. Fendler, Adv. Mater. 16, 1685 (2004)

2. N. Felidj, J. Grand, G. Laurent, J. Aubard, G. Levi, A. Hohenau, N. Galler, F.R. Aussenegg, J.R. Krenn, J. Chem. Phys. 128, 094702 (2008)

3. J. Nelayah, M. Kociak, O. Stéphan, N. Geuquet, L. Henrard, F.J. Garcìa de Abajo, I. Pastoriza-Santos, L.M. Liz-Marzàn, C. Colliex, Nano Lett. 10, 902 (2010)

4. L. Douillard, F. Charra, Z. Korczak, R. Bachelot, S. Kostcheev, G. Lerondel, P.M. Adam, P. Royer, Nano Lett. 8, 935 (2008)

5. L. Novotny, Phys. Rev. Lett. 98, 266802 (2007)

6. G. Boudarham, N. Feth, V. Myroshnychenko, S. Linden, J. García de Abajo, M. Wegener, M. Kociak, Phys. Rev. Lett. 105, 255501 (2010)

7. F.J.G. de Abajo, A. Howie, Phys. Rev. B 65, 115418 (2002)

8. L.J. Sherry, S.H. Chang, G.C. Schatz, R.P. Van Duyne, B.J. Wiley, Y.N. Xia, Nano Lett. 5, 2034 (2005)

9. J. Aaron, E. de la Rosa, K. Travis, N. Harrison, J. Burt, M. José-Yacamán, K. Sokolov, Opt. Express 16, 2153 (2008)

10. L. Rodríguez-Lorenzo, R.A. Álvarez-Puebla, I. Pastoriza-Santos, S. Mazzucco, O. Stéphan, M. Kociak, L.M. Liz-Marzán, F.J. García de Abajo, J. Am. Chem. Soc. 131, 4616 (2009)

11. E. Hao, R.C. Bailey, G.C. Schatz, J.T. Hupp, S.Y. Li, Nano Lett. 4, 327 (2004)

12. M. Yamamoto, Y. Kashiwagi, T. Sakata, H. Mori, M. Nakamoto, Chem. Mater. 17, 5391 (2005)

13. X. Zou, E. Ying, S. Dong, Nanotechnology 17, 4758 (2006)

14. P.S. Kumar, I. Pastoriza-Santos, B. Rodríguez-González, F.J. García de Abajo, L.M. Liz-Marzán, Nanotechnology 19, 015606 (2008)
15. C.L. Nehl, H.W. Liao, J.H. Hafner, Nano Lett. 6, 683 (2006)

16. F. Hao, C.L. Nehl, J.H. Hafner, P. Nordlander, Nano Lett. 7, 729 (2007)

17. F.J.G. de Abajo, M. Kociak, Phys. Rev. Lett. 100, 106804 (2008)

18. Z.L. Wang, Micron 27, 265 (1996)

19. A. Rivacoba, N. Zabala, J. Aizpurua, Prog. Surf. Sci. 65, $1(2000)$

20. D. Ugarte, C. Colliex, P. Trebbia, Phys. Rev. B 45, $4332(1992)$

21. L.A. Bursill, P.A. Stadelmann, J.L. Peng, S. Prawer, Phys. Rev. B 49, 2882 (1994)

22. M. Kociak, L. Henrard, O. Stéphan, K. Suenaga, C. Colliex, Phys. Rev. B 61, 13936 (2000)

23. T. Stockli, J.M. Bonard, A. Chatelain, Z.L. Wang, P. Stadelmann, Physica B 280, 4844 (2000)

24. O. Stephan, M. Kociak, L. Henrard, K. Suenaga, A. Gloter, M. Tence, E. Sandre, C. Colliex, J. Electron Spectrosc. Relat. Phenomena 114, 209 (2001)

25. D. Taverna, M. Kociak, V. Charbois, L. Henrard, O. Stephan, C. Colliex, J. Electron Spectrosc. Relat. Phenomena 129, 293 (2003)

26. R. Arenal, O. Stephan, M. Kociak, D. Taverna, A. Loiseau, C. Colliex, Phys. Rev. Lett. 95, 127601 (2005)

27. A. Seepujak, U. Bangert, A.J. Harvey, P.M.F.J. Costa, M.L.H. Green, Phys. Rev. B 74, 075402 (2006)

28. P. Moreau, N. Brun, C.A. Walsh, C. Colliex, A. Howie, Phys. Rev. B 56, 6774 (1997)

29. M. Couillard, M. Kociak, O. Stephan, G.A. Botton, C. Colliex, Phys. Rev. B 76, 165131 (2007)

30. M. Couillard, A. Yurtsever, D.A. Muller, Phys. Rev. B 77, 085318 (2008)

31. T. Eberlein, U. Bangert, R.R. Nair, R. Jones, M. Gass, A.L. Bleloch, K.S. Novoselov, A. Geim, P.R. Briddon, Phys. Rev. B 77, 233406 (2008)

32. F.J. García de Abajo, J. Aizpurua, Phys. Rev. B 56, 15873 (1997)

33. F.J. García de Abajo, A. Howie, Phys. Rev. Lett. 80, 5180 (1998)

34. J. Aizpurua, A. Howie, F.J. García de Abajo, Phys. Rev. B 60, 11149 (1999)

35. M. Bosman, V.J. Keast, M. Watanabe, A.I. Maaroof, M.B. Cortie, Nanotechnology 18, 165505 (2007)

36. J. Nelayah, M. Kociak, O. Stephan, F. Javier García de Abajo, M. Tence, L. Henrard, D. Taverna, I. PastorizaSantos, L.M. Liz-Marzán, C. Colliex, Nat. Phys. 3, 348 (2007)

37. C. Jeanguillaume, C. Colliex, Ultramicroscopy 28, 252 (1989)

38. G. Kothleitner, F. Hofer, Micron 34, 211 (2003)

39. A. Gloter, A. Douiri, M. Tence, C. Colliex, Ultramicroscopy 96, 385 (2003)

40. L.F. Zagonel, S. Mazzucco, M. Tencé, K. March, R. Bernard, B. Laslier, G. Jacopin, M. Tchernycheva, L. Rigutti, F.H. Julien, R. Songmuang, M. Kociak, Nano Lett. 11, 568 (2011)

41. The EELSLL code is freely available on the Orsay STEM group website http://www.lps.u-psud.fr/collectif/gr_27

42. E.D. Palik, Handbook of Optical-Constants, Vol. 1 (Academic Press, 1984)

43. P.B. Johnson, R.W. Christy, Phys. Rev. B 6, 4370 (1972) 